\title{
Bilateral upper lobe pulmonary edema during gynecologic laparoscopic surgery in the Trendelenberg position
} -A case report-

\author{
Jae-Hang Shim, Woo Jong Shin, and Sang Hoon Lee \\ Department of Anesthesiology and Pain Medicine, Hanyang University College of Medicine, Seoul, Korea
}

\begin{abstract}
A 25-year-old woman was diagnosed with a ruptured ectopic pregnancy. During laparoscopic surgery, the patient was in the Trendelenberg position $\left(20^{\circ}\right.$ degrees). Massive froth in the endotracheal tube was observed at the end of surgery. A portable chest x-ray, checked at the end of the operation, showed diffuse haziness in both upper lung fields. After one hour of aggressive treatment with drugs and positive mechanical ventilation, the amount of froth in the endotracheal tube was reduced considerably. Considering the symptom and radiologic findings, we concluded that diffuse bilateral upper lung field haziness was due to atypical pulmonary edema. We speculated that the rapid improvement of pulmonary edema was due to redistribution of fluid to the lowest part of lung by immediate reversing the patient's Trendelenberg position, along with aggressive treatment. (Korean J Anesthesiol 2010; 59:
\end{abstract} S163-S166)

Key Words: Laparoscopic surgery, Trendelenberg position, Upper lobe pulmonary edema.

Perioperative pulmonary edema (PE) causes body fluids to extrude from pulmonary arterioles and capillaries into interstitial tissue and alveoli. This results in life threatening symptoms of hypoxia. Common causes of typical PE include heart failure, aspiration, trauma, and erythrocytosis, while causes of atypical PE are upper respiratory obstruction, sudden reexpansion of a collapsed lung, low serum albumin levels, changes in diffusion gradient or gravitational factors of arterioles, extruding pleural effusion, and laparoscopy [1]. Diagnosis is difficult because it can result from a sole etiologic factor or as a complicated combination of more than two. Early diagnosis can also prove to be difficult, especially in atypical cases. We report a case of a young healthy woman with bilateral upper lobe PE seen during laparoscopic surgery in the Trendelenberg position.

\section{Case Report}

A 25-year-old woman weighing $56 \mathrm{~kg}$ and with a height of $154 \mathrm{~cm}$ was diagnosed with a ruptured ectopic pregnancy, revealing a hemoglobin level of $7.4 \mathrm{~g} / \mathrm{dl}$ and hematocrit of $21.5 \%$ at admission. The patient's chest x-rays were normal (Fig. 1). Anesthesia was induced with ketamine and rocuronium and maintained with $\mathrm{N}_{2} \mathrm{O}-\mathrm{O}_{2}$-sevoflurane. $\mathrm{CO}_{2}$ insufflation was

Received: February 2, 2010. Revised: 1st, February 23, 2010; 2nd, March 22, 2010. Accepted: April 23, 2010.

Corresponding author: Woo Jong Shin, M.D., Department of Anesthesiology and Pain Medicine, Hanyang University College of Medicine, Guri Hospital, Gyomun-dong, Guri 471-020, Korea. Tel: 82-31-560-2390, Fax: 82-31-563-1731, E-mail: swj0208@hanyang.ac.kr

(C) This is an open-access article distributed under the terms of the Creative Commons Attribution Non-Commercial License (http:// creativecommons.org/licenses/by-nc/3.0/), which permits unrestricted non-commercial use, distribution, and reproduction in any medium, provided the original work is properly cited. 


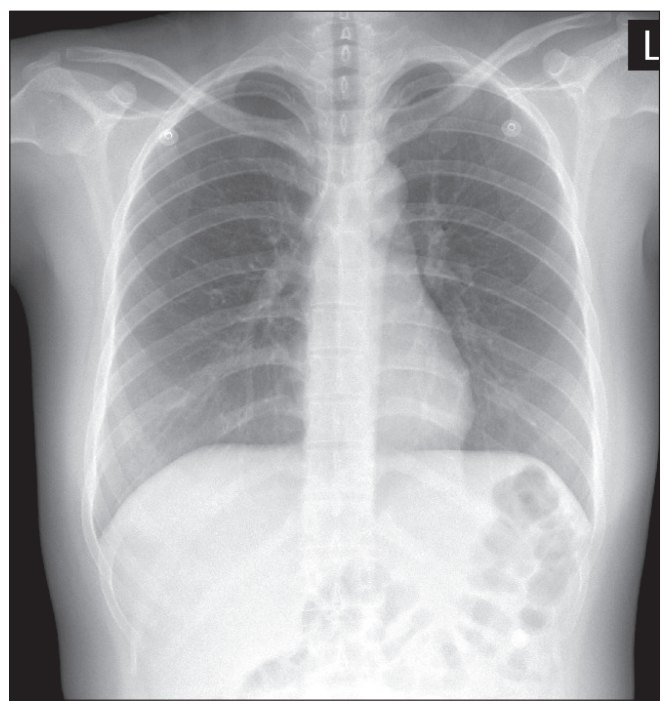

Fig. 1. Preoperative chest posteroanterior (PA) shows no active lesion in the lung.

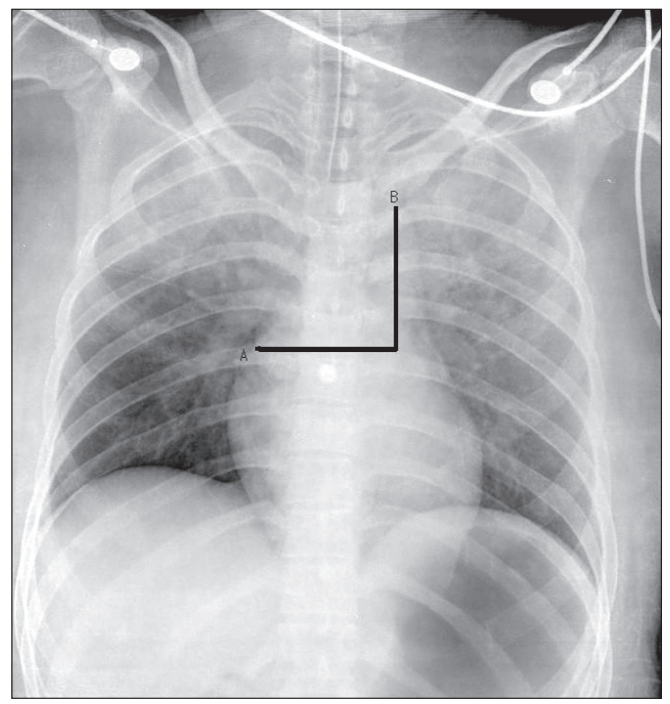

Fig. 2. Postoperative chest anteroposterior (AP), checked at the end of surgery, shows diffuse haziness in both upper lung fields. Vascular pedicle width (VPW), calculated by (A) measuring across to the point at which the superior vena cava crosses the right mainstem bronchus and (B) dropping a perpendicular line from the point at which the left subclavian artery exits the aortic arch, is greater than $70 \mathrm{~mm}$.

performed and the patient was tilted to $20^{\circ}$ Trendelenberg position. After intravenous infusion of 3 units of packed red blood cells (PRBC), 1,250 $\mathrm{ml}$ of crystalloid, and $500 \mathrm{ml}$ of colloid solution over a period of 50 minutes, at a $\mathrm{FiO}_{2}$ of 0.5 , arterial blood gas analysis (ABGA) showed a pH of 7.31, $\mathrm{PaCO}_{2}$ of 39.3 $\mathrm{mmHg}, \mathrm{PaO}_{2}$ of $37.3 \mathrm{mmHg}$, base excess of -6.5 , hemoglobin level of $8.7 \mathrm{~g} / \mathrm{dl}$, and hematocrit $25 \%$. After completion of the operation, delivery of anesthetics was stopped and $100 \%$ oxygen was given, followed by reversal of muscle relaxation.

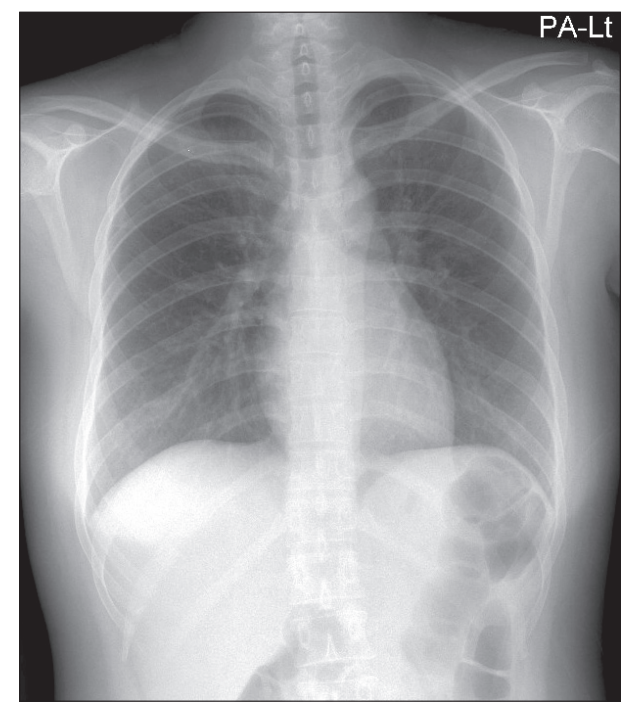

Fig. 3. Postoperative chest posteroanterior (PA) checked at postoperative day one, does not show diffuse haziness in both upper lung fields but shows pulmonary vascular blurring in the whole lung field.

Before extubation, the patient showed spasmodic coughing and pink frothy sputum with extrusion of large amounts of foamy fluid. A chest x-ray was immediately taken revealing diffuse haziness over both upper lung fields (Fig. 2). The patient was immediately shifted to the $15^{\circ}$ head up position and aggressive treatment with continuous positive pressure ventilation combined with furosemide was initiated. Approximately 1 hour later, $1,250 \mathrm{ml}$ of urine was excreted and ABGA showed a $\mathrm{pH}$ of 7.27, $\mathrm{PaCO}_{2}$ of $44 \mathrm{mmHg}, \mathrm{PaO}_{2}$ of $379 \mathrm{mmHg}$, base excess of -6.8 at a $\mathrm{FiO}_{2}$ of 1.0. After tracheal reflexes and respiratory functions returned to normal, the patient was able to reach tidal volume of greater than $8 \mathrm{ml} / \mathrm{kg}$ and was consequently extubated. In the recovery room, the patient was maintained in a head up position. ABGA showed $\mathrm{PaO}_{2}$ of $147.6 \mathrm{mmHg}$, hemoblogin level of $15.2 \mathrm{~g} / \mathrm{dl}$ at $35 \%$ venturi mask oxygen. The haziness seen previously in both upper lung fields disappeared in the first postoperative chest x-ray (Fig. 3).

\section{Discussion}

There are two clinical classifications of PE, cardiac and noncardiac [1]. Milne et al. [2] stated that $90 \%$ of distributed fluid in cardiac PE was seen diffusely throughout the lungs, while in noncardiac PE, caused by fluid overload, $70 \%$ was seen in the hilar area and $45 \%$ of permeability PE occurred in the periphery of the lung. In our case, haziness occurred in both upper lung fields, showing a different distribution. Ely and Haponik [3] mentioned vascular pedicle width (VPW) during PE. VPW was calculated by measuring across to the point at which the superior vena cava crosses the right mainstem bronchus and dropping 
a perpendicular line from the point at which the left subclavian artery exits the aortic arch (Fig. 2). They claimed that if the value of VPW is less than $43 \mathrm{~mm}$, the possibility of an injury pattern of edema was high, and if the value of VPW was greater than 53 $\mathrm{mm}$ with a high blood volume, the possibility of heart or renal failure would be high. In cardiac PE, haziness over the vascular pedicle increases in $60 \%$ of patients. In noncardiac $P E$, haziness increases in $85 \%$ [2]. Although there are minor variations in the value of VPW among each evaluator, VPW was greater than $70 \mathrm{~mm}$ in our case, which was predictive of a volume overload state (Fig. 2). Taken together, noncardiac PE was suspected. Milne et al. [2] has pointed out that pulmonary blood flow distribution were usually inverted and peribronchial cuffs, pleural effusion were quite frequently observed on chest $\mathrm{x}$-rays in cardiac PE. The reason for these characteristics is that fluid initially accumulates in the soft connective tissue around vessels and bronchus in cardiac PE. In contrast, radiologic findings in hydrostatic PE, frequently show fluid accumulation around the bronchioles and subjective cardiomegaly, increase in VPW, and pleural effusion. In hydrostatic PE, fluid overload causes extravascular fluid to shift to the lower osmotic pressure areas of the lung in the central area, thus, sending the fluid to the center and creating an enhanced shadow. Furthermore, in young patients, where lung compliance is good and cardiac diseases are few, fluid would more likely accumulate in the central areas. In our case, hilar abnormalities, enhanced interlobular septal lines, peribronchial cuffs, and air bronchograms were not seen.

Arieff [4] stated that there is a high risk for severe PE when greater than $6 \mathrm{~L}$ or $67 \mathrm{ml} / \mathrm{kg}$ of intravenous fluid is given perioperatively. In our patient, three units of PRBC, 1,250 ml of crystalloid and $500 \mathrm{ml}$ of colloid solution were given within 100 minutes during the operation. One hundred $\mathrm{ml}$ of urine was excreted and estimated blood loss was approximately $1,000 \mathrm{ml}$; consequently, 1,000 ml of fluid were overloaded. Despite the young age of the patient, short operation time and mild fluid overloading, severe PE developed. We believe this is the result of the patient being in the Trendelenberg position causing an increase in the hydrostatic pressure and leading to a redistribution of fluids within the vasculature to both upper lobes. This is compatible with the radiological findings of haziness seen in both upper lobes. Atypical findings of PE seen in only one lung or in a specific area can be found in various conditions, such as in the effect of gravity $[5,6]$ or changes in pulmonary perfusion $[5,7]$, pulmonary venous circulation $[5,7]$, intrapleural pressure [5], or lung ventilation [5]. PE occurring in single or multiple areas on chest $\mathrm{x}$-rays may be seen in infective pneumonia, acute interstitial pneumonitis, and acute respiratory distress syndrome (ARDS). Furthermore, consolidation seen in the upper lobes can be interpreted as tuberculosis [8]. Important factors greatly influencing the distribution of PE are pulmonary circulation and capillary hydrostatic pressure [6,9].

In our patient, the length from the apex to the base of the lung measured $30 \mathrm{~cm}$ (equivalent to $22 \mathrm{mmHg}$ ). In the Trendelenberg position, there is an approximately $10 \mathrm{~cm}$ (equivalent to $7.3 \mathrm{mmHg}$ ) difference in the length from the apex to the base. In a study done by Denison et al. [10], the ratio of blood flow of the apex to the base of the lung in a healthy subject was $1: 2.5$, but in an inverted position the ratio changed to $1.73: 1$. Zimmerman et al. [9] demonstrated that in a gravitational shift test, $\mathrm{x}$-rays taken $2-3$ hours after maintaining a supine, semi-sitting, or lateral position showed, PE in 10 of 12 cases; furthermore, in 6 of 7 cases of infection plus PE, migration of fluids could be seen. It is evident that gravity had a major influence on the pulmonary blood flow distribution and capillary hydrostatic pressure, suggesting an important role of gravity in the distribution of PE. We believe this is enough to support the idea that $2-3$ hours of position change can result in a redistribution of PE. In addition, the time elapsed may have had an influence on the redistribution of PE. Complete disappearance of the infiltration seen several hours later is evidence that gravitational forces must have had an influence on pulmonary circulation. PE is seen in less than $1 \%$ of laparoscopic hysterectomies, which usually occurs after fluid intake of over 5-7 liters [4]. Thus, during obstetric surgery, parameters that can measure total amount of baseline flow of fluids, such as central venous pressure, are needed.

PE resulting from transfusion can be difficult to detect and may result in severe complications, such as transfusion-related acute lung injury (TRALI) [11] and transfusion-associated circulatory overload (TACO). Classification of the two is not easy, as the adverse effects of transfusion in TACO have not been established and reports of it occurring are equivocal and range from below $1 \%$ up to $8 \%$ [12]. There was mild fluid overload in our patient. An improvement of PE and hypoxia by diuretics does not match the criteria for TRALI. We believe that the rise in hemoglobin level seen in the follow-up ABGA was due to the use of diuretics concentrating the blood. Although we did not perform any tests for the possible adverse effects of the overloading of blood, $\mathrm{x}$-ray findings and increase in VPW were inferred as resulting from hydrostatic PE.

We report a case of sudden onset of noncardiac PE developed after mild fluid and transfusion overloading, with simultaneous redistribution in a young patient without a history of heart problem under laparoscopic surgery. Intra- and postoperative ABGA presented severe hypoxia; however, after diagnosing the patient's condition even after misinterpretation of an ABGA as a venous sampling, aggressive and immediate treatment was successful. Redistribution related to changing the patient's position allowed for recovery and extubation in a relatively 
short time. For a more accurate differential diagnosis, tests other than chest $\mathrm{x}$-rays, such as PE fluid protein concentration ratio and echocardiography, are needed. Unfortunately, such studies were not performed.

If chest x-rays present findings similar to pneumonia or ARDS in both upper lobes after laparoscopic surgery in the Trendelenberg position, one must consider the possibility of PE and the patient should be immediately moved to the head up position to allow redistribution of fluids. With aggressive treatment according to etiology, one can achieve good results and eventual recovery of patients.

\section{References}

1. Gluecker T, Capasso P, Schnyder P, Gudinchet F, Schaller MD, Revelly JP, et al. Clinical and radiologic features of pulmonary edema. Radiographics 1999; 19: 1507-31.

2. Milne EN, Pistolesi M, Miniati M, Giuntini C. The radiologic distinction of cardiogenic and noncardiogenic edema. AJR Am J Roentgenol 1985; 144: 879-94.

3. Ely EW, Haponik EF. Using the chest radiograph to determine intravascular volume status: the role of vascular pedicle width. Chest 2002; 121: 942-50.
4. Arieff AI. Fatal postoperative pulmonary edema: pathogenesis and literature review. Chest 1999; 115: 1371-7.

5. Alarcon JJ, Guembe P, de Miguel E, Gordillo I, Abellás A. Localized right upper lobe edema. Chest 1995; 107: 274-6.

6. Mehta JB, Mack L, Raetz D. Acute bilateral upper lobe infiltrate in a young man. Chest 1978; 73: 861-2.

7. Lee SW, Kim MW. Acute pulmonary edema progressing from unilateral to bilateral after brief laparoscopic surgery under lateral position in elderly patient. Korean J Anesthesiol 2008; 55: 644-7.

8. Kroegel C, Reibetaig A, Hengst U, Mock B, Häfner D, Grahmann PR. Bilateral symmetrical upper-lobe opacities: an unusual presentation of bronchiolitis obliterans organizing pneumonia. Chest 2000; 118: 863-5.

9. Zimmerman JE, Goodman LR, St Andre AC, Wyman AC. Radiographic detection of mobilizable lung water: the gravitational shift test. AJR Am J Roentgenol 1982; 138: 59-64.

10. Denison D, Ernsting J, Frayer D. The effect of the inverted posture upon the distribution of pulmonary blood flow in man. J Physiol 1964; 172: 49-50.

11. Triulzi DJ. Transfusion-related acute lung injury: current concepts for the clinician. Anesth Analg 2009; 108: 770-6.

12. Gajic O, Gropper MA, Hubmayr RD. Pulmonary edema after transfusion: how to differentiate transfusion-associated circulatory overload from transfusion-related acute lung injury. Crit Care Med 2006; 34: S109-13. 